\title{
Impacto en el acceso a medicamentos en la población colombiana después de la actualización del plan de beneficios en el $2012^{*}$
}

\author{
Impact on the Access to Medication in Colombian \\ Population after the Benefit Plan Update in 2012
}

\section{Impacto no acesso a remédios na população colombiana após a atualização do plano de benefícios no 2012}

Fecha de recepción: 20-06-14 Fecha de aceptación: 09-09-14 Disponible en línea: 01-07-14 doi: 10.11144/Javeriana.rgyps13-27.iamp

Cómo citar este artículo:

Romero-Prada M, Marrugo-Figueroa R, Acero G, Arango-Bautista C, Alvis-Guzmán, N. Impacto en el acceso a medicamentos en la población colombiana después de la actualización del plan de beneficios en el 2012. Rev. Gerenc. Polít. Salud. 2014; 13(27): 228-241. http://dx.doi.org/10.11144/Javeriana.rgyps13-27.iamp

\author{
Martín Romero-Prada** \\ Rubén Marrugo-Figueroa*** \\ Germán Acero**** \\ Carlos Arango-Bautista***** \\ Nelson Alvis-Guzmán******
}

\footnotetext{
Artículo de investigación, nombre del proyecto: Impacto en el acceso a medicamentos en la población colombiana posterior a la actualización del plan de beneficios 2012, entidad financiadora: Fundación Salutia - Centro de Investigaciones en Economía, Gestión y Tecnologías en Salud. Proyecto desarrollado desde octubre del 2012 hasta junio del 2014. Este trabajo fue presentado como póster con resultados preliminares en el evento Ispor 4th Latin American Conference, realizado en Buenos Aires, Argentina, del 12 al 14 de septiembre del año 2013. Por la presentación en dicho evento, el resumen fue publicado en Value In Health. La referencia de este trabajo es la siguiente: Romero M, Acero G, Marrugo R, Alvis N, Arango C. Análisis del impacto en la demanda de tecnologías incluidas en el plan de beneficios colombiano (POs) posterior a su actualización en 2012 [resumen]. Value in Health. 2013; 16(Issue 7):A670. DOI: 10.1016/j.jval.2013.08.1939. También fue aceptado y presentado como ponencia en el 3er Congreso de la Asociación Colombiana de Economía de la Salud, en la ciudad de Medellín, Colombia, del 20 al 22 de noviembre de 2013. Este estudio fue financiado por la Fundación Salutia - Centro de Investigaciones en Economía, Gestión y Tecnologías en Salud.

** Candidato a doctor en Salud Pública de la Universidad Nacional de Colombia, codirector de la Fundación Salutia - Centro de Investigaciones en Economía, Gestión y Tecnologías en Salud, Carrera 71B, núm. 116A-12, Bogotá, Colombia. Tel: (57) 1 613-4609, Fax: (57) 1 617-9133. Correo electrónico: martin.romero@salutia.org

*** $\quad$ Máster en Economía de la Universidad Externado de Colombia, investigador de la Fundación Salutia - Centro de Investigaciones en Economía, Gestión y Tecnologías en Salud, Bogotá, Colombia. Correo electrónico: ruben. marrugo@salutia.org

***** Profesional en Finanzas y Relaciones Internacionales de la Fundación Universitaria Monserrate, investigador de la Fundación Salutia - Centro de Investigaciones en Economía, Gestión y Tecnologías en Salud. Correo electrónico: german.acero@salutia.org

****** Candidato a doctor en Salud Pública de la Universidad Nacional de Colombia, codirector de la Fundación Salutia - Centro de Investigaciones en Economía, Gestión y Tecnologías en Salud. Correo electrónico: arango.carlosh@salutia.org

****** Doctor en Economía y Gestión de la Salud de la Universidad Politécnica de Valencia (España), director del Grupo de Investigación en Economía y Gestión de la Salud, docente de la Universidad de Cartagena. Correo electrónico: nalvis@yahoo.com
} 


\section{Resumen}

Objetivo: evaluar los cambios en la demanda de servicios en salud, luego de la actualización del plan de beneficios de Colombia, entre los años 2010 y 2012. Métodos: estudio tipo descriptivo y explicativo, donde se evalúa el uso de tecnologías sanitarias antes y después de la actualización del Plan Obligatorio de Salud. Se tomaron las dispensaciones de medicamentos entre el 2010 y el 2012 del Sistema de Información de Precios de Medicamentos. Para valorar los cambios se construyó una escala de valoración de cambios porcentuales. Resultados: se evidenciaron cambios en el comportamiento de la demanda de servicios. Como se esperaba, la actualización del Plan de Beneficios representa para los pacientes mayor acceso a las nuevas tecnologías, medido a través del mayor uso de tecnologías nuevas. Conclusión: la actualización del plan de beneficios mejoró el acceso de los pacientes a una gran parte de los servicios de salud.

Palabras clave: sistema de salud; Plan de Beneficios; POs; precios de medicamentos; acceso al sistema de salud; Sismed

\section{Abstract}

Objective: Assessing the changes in the demand of health services after the update of the benefit plan in Colombia, during 2010 and 2012. Methods: Descriptive and Explanatory type study, where the use of healthcare technologies before and after the update of the Compulsory Health Plan is assessed. We took the drug dispensations between 2010 and 2012 from the Drug Price Information System. To assess the changes, we made an evaluation scale of percentage changes. Results: We found evidence of changes in the behavior of the demand of services. As expected, the update of the Benefits Plan represents greater access to new technologies, measured through a greater use of new technologies. Conclusion: The update of the Benefits Plan improved access for patients to a large part of the health services.

Keywords: health system; benefits plan; POS; drug prices; access to the health system; Sismed

\section{Resumo}

Objetivo: avaliar as mudanças na demanda de serviços em saúde, após atualização do plano de benefícios da Colômbia, entre os anos 2010 e 2012. Métodos: estudo tipo descritivo e explicativo, onde avalia-se o uso de tecnologias sanitárias antes e depois da atualização do Plano Obrigatório de Saúde. Tomaram-se as dispensações de medicamentos entre o 2010 e 2012 do Sistema de Informação de Preços de Medicamentos. Para avaliar as mudanças foi construída uma escala de valoração de mudanças porcentuais. Resultados: evidenciaram-se mudanças no comportamento da demanda de serviços. Como esperado, a atualização do Plano de Benefícios representa para os pacientes maior acesso às novas tecnologias, medido a través do maior uso de tecnologia nova. Conclusão: a atualização do plano de benefícios melhorou o acesso dos pacientes a uma grande parte dos serviços de saúde.

Palavras-chave: sistema de saúde; Plano de Benefícios; pos; preços de remédios; acesso ao sistema de saúde; Sismed 


\section{Introducción}

Mediante la Ley 100 de 1993, Colombia reformó su modelo de sistema de salud, con lo cual logró pasar de un modelo segmentado a uno de pluralismo estructurado (1), denominado Sistema General de Seguridad Social en Salud (sGSss), con atención administrada y competencia regulada (2).

Este nuevo sistema busca asegurar a toda la población mediante dos regímenes. El primero es el contributivo y es para trabajadores o personas con capacidad de pago, las cuales se afilian a una aseguradora (Empresa Promotora de Salud - EPS contributiva) mediante el pago de un aporte basado en el salario, que se acumula en un fondo único (Fondo de Solidaridad y Garantía - Fosyga). Con ello, el cotizante y sus beneficiarios adquieren el derecho a recibir un plan de beneficios de servicios en salud (Plan Obligatorio de Salud - POS), que incluye un listado de tecnologías establecidas por el Ministerio de Salud y Protección Social. El segundo régimen es el subsidiado, financiado por el Estado y administrado por los entes territoriales. Este tiene como propósito garantizarle a la población pobre y vulnerable, previamente identificada y seleccionada mediante un Sistema de Identificación de Beneficiarios de Subsidios (Sisben), el derecho a la salud mediante la afiliación a una aseguradora (EPS-subsidiada).

Las EPs, tanto contributivas como subsidiadas, reciben del Fosyga y de los entes territoriales una prima de riesgo (unidad de pago por capitación - UPC) por cada afiliado (cotizante y/o beneficiarios), para garantizarle a los usuarios el acceso al pos (3).

El pos es planteado en la Ley 100 como una lista positiva, tanto de medicamentos como de servicios de salud, que debería actualizarse periódicamente, de acuerdo con los cambios en la estructura demográfica de la población, el perfil epidemiológico nacional, la tecnología apropiada disponible en el país y las condiciones financieras del sistema. Sin embargo, los procesos de actualización del POS que permiten la incorporación de nuevas tecnologías en salud (NTS) no siguieron la regularidad esperada.

Es proceso de actualización del pos ha sido mediado por decisiones de por lo menos cuatro instituciones desde 1993: 1) el Consejo Nacional de Seguridad Social en Salud (CNSSS), destinado por la Ley 100 de 1993 como la institución responsable de decidir cuáles eran los servicios de salud que se incorporaban o retiraban del Pos; 2) los jueces de la República, que intentando proteger el derecho a la salud, obligan al Estado y/o a los aseguradores a cumplir decisiones médicas en las que se brindan NTS; 3) los comités técnico-científicos (СтC) de las EPS, que identifican la pertinencia y racionalidad científica de las NTS (4); y 4) la Comisión de Regulación en Salud (CRES), que según la Ley 1122 de 2007 reemplaza al cNsss y adquiere la responsabilidad de realizar los estudios técnicos para evaluar la incorporación de NTS al POs.

De esta manera, en el 2007, ante la precaria gestión del cNSSS para actualizar el POS, y frente a la creciente actuación de los jueces, la Corte Constitucional de Colombia expidió la Sentencia T-760, en la que obliga al Gobierno a asegurar el acceso a los servicios de salud y unificar los derechos de los afiliados al sGSss respecto al acceso al POS.

No obstante, el número de tutelas se incrementó exponencialmente, bajo la consigna del derecho a la salud, solicitando diferentes tecnologías que están o no dentro del pos $(5,6)$. Es en este punto donde radica uno de los principales problemas del acceso integral al sistema de salud por parte de 
los colombianos. A pesar de que la Ley 100 obliga al Estado colombiano a aumentar el plan de beneficios en salud, de acuerdo con las necesidades de los ciudadanos, esto se ha realizado a medias, afectando directamente a los colombianos que pretenden acceder al sistema. Sin embargo, no fue sino hasta el 2011, y finales del 2012, que la CRES realizó un proceso de actualización del pos para incluir NTS, las cuales fueron analizadas en términos de efectividad, seguridad e impacto presupuestal en el sistema de salud, comparándolas con las opciones disponibles en el POS de ese entonces.

La prioridad para la actualización del pos se centró en las tecnologías más recobradas, ya que de esta manera se disminuiría el recobro al Fosyga y dichas tecnologías serían asumidas por las EPs dentro del presupuesto concebido por UPC. Finalmente, luego de un análisis de suficiencia presupuestal, se emitió una nueva lista con 59 nuevas tecnologías y cerca de 120 nuevas indicaciones y concentraciones de medicamentos, la cual se institucionalizó por medio del Acuerdo 29 del 2011 de la CRES (7).

No obstante, no existe un análisis profundo sobre si dichas tecnologías son altamente necesarias para el sistema. Es por esto que la investigación sobre los impactos de esta actualización se convierte en una herramienta de gran importancia por los siguientes dos motivos: 1) saber si las tecnologías nuevas son necesarias o si por el contrario los pacientes no las están demandando, 2) en caso de que estén siendo demandadas, cuáles son las tecnologías y enfermedades a las que el sistema les debería prestar más atención.

Aunque el proceso fue desarrollado de acuerdo con los lineamientos definidos por la CRES, es importante evaluar cuál fue el cambio real en el acceso a estas nuevas tecnologías por parte de la población colombiana.
Esto debido, principalmente, a que más allá de la norma de incorporación de tecnologías al plan de beneficios, todavía existen barreras de acceso generadas por las EPS, en razón de las preferencias de otras alternativas menos efectivas, por su bajo costo o por la existencia de otras alternativas innovadoras que podrían ser objeto de recobro.

Por lo anterior, el objetivo de la presente investigación es evaluar las variaciones en la demanda atendida de tecnologías, luego de la actualización del pos en el año 2012, como indicador indirecto del acceso a los servicios de salud. De esta manera, se podrá determinar en términos de consumo de tecnologías, si la actualización del pos surtió un efecto positivo sobre los colombianos, en materia de acceso a tecnologías nuevas.

\section{Metodología}

Este estudio descriptivo y explicativo realizó un análisis comparativo de demanda atendida antes y después de la actualización del pos. Aunque la actualización se realizó para medicamentos, dispositivos médicos y procedimientos, este análisis tuvo como único criterio de inclusión la selección de los medicamentos incluidos en el Acuerdo 29 del 2011 (7), del cual se tomó una muestra a través de un aleatorio simple: 20 tecnologías (37\% del total).

Para cada una de las tecnologías seleccionadas se identificaron sus comparadores en el POS, utilizando como referencia los análisis realizados por la CRES para tomar la decisión de incorporación.

Como fuente de datos se utilizó el Sistema de Información de Precios de Medicamentos (Sismed), el cual es un registro estandarizado y normalizado de carácter oficial que depende del Ministerio de Comercio de Colombia, 
quien analiza y controla el comportamiento de los precios de los medicamentos en el país y orienta la regulación del mercado. Para ello registra el almacenamiento, flujo, transferencia y disposición de la información del mercado de medicamentos en la cadena de producción y distribución (8). En este sistema los diferentes actores del mercado de medicamentos reportan cantidades comercializadas y precio de la transacción en diferentes ámbitos del mercado.

De cada uno de los medicamentos seleccionados se recogió información sobre sus unidades despachadas, registradas para los años 2010 y 2011 (antes de la actualización del Pos), y el 2012 como año de evaluación de la actualización del pos (el cual será denominado como "nuevo pos" para efectos prácticos de esta investigación). La fuente de información (el Sismed) realiza diferenciación en la dispensación de medicamentos, dependiendo del canal de distribución, sea comercial o institucional. Por ende, se analizó el comportamiento de las tecnologías en los dos canales de distribución, en donde el primero se refiere a las unidades despachadas al público en general, en droguerías y grandes superficies, y el segundo a las unidades despachadas dentro del sistema de salud, considerando hospitales y EPS.

El acceso a los servicios de salud fue medido no solo como el valor total absoluto que podría significar un mejor acceso, sino que además se midió en las variaciones dentro de los diferentes canales. Los datos fueron analizados en términos de cambios porcentuales entre periodos y expresados en pesos del 2013.

A la hora de medir el consumo de medicamentos, es importante tener tanto un sistema de clasificación como una unidad de medida. Por ello, para homogenizar los problemas de dosis, presentaciones y concentraciones de medicamentos en el análisis, aparece la unidad de medida técnica llamada dosis diaria definida (DDD), que se utiliza en los estudios de consumo de medicamentos. Luego de identificar las unidades despachadas de cada tecnología, determinando su forma de presentación (pastillas, ampollas, blíster, bolsa, etc.), se consolida bajo la medida estandarizada de DDD $^{1}$.

Como unidad de medida podría utilizarse el envase o la presentación de las tecnologías, pero surge el inconveniente de que no todos los países tienen la misma dosificación para un mismo medicamento. Además, puede haber diferencias sustanciales para un mismo principio activo en la dosificación que presentan las diferentes marcas comerciales. Por otra parte, la posología utilizada está sujeta a variaciones dependientes del propio médico, de la gravedad del paciente o incluso de razones de mercadeo difícilmente justificables.

De esta manera, para el análisis del comportamiento de las tecnologías, posteriormente a la cuantificación de las unidades despachadas, se realizan los ajustes por DDD y se desarrollan los análisis vía estimación de variaciones porcentuales, de la siguiente manera:

$$
\Delta T E C=\frac{\text { Cant.final-Cant.Inicial }}{\text { Cant.Inicial }} * 100=\mathrm{X} \%
$$

En el caso específico de imatinib, el cambio porcentual de la dispensación de una tecnología del año 2011 al 2012 se estima de la siguiente forma:

1 La DDD es una unidad técnica de medida que se define como la dosis media diaria de mantenimiento de un medicamento cuando se usa rutinariamente en su principal indicación, por una vía de administración determinada y a veces con una concentración dada, expresándose en cantidad de principio activo (por ejemplo, unidades internacionales, miligramos, etc.). Estas DDD han sido escogidas de acuerdo con la literatura biomédica y al asesoramiento de un número de clínicos expertos en este campo. 


$$
\Delta \text { Imatinib }=\frac{\text { Cant2012-Cant } .2011}{\text { Cant.2011 }} * 100=X \%
$$

Luego de consolidar las unidades dispensadas de las presentaciones evaluadas de 100 mg y $400 \mathrm{mg}$, y tras realizar el ajuste por DDD, se estimó la variación porcentual que se presenta de un año a otro. El escenario ideal sería el número de pacientes por patología, determinando su consumo; sin embargo, no fue posible establecer el número de pacientes indicados con cada tecnología, por cuanto el registro Sismed no lo incluye, y aunque existen tecnologías que pueden ser usadas en varias patologías, tampoco es posible realizar esta discriminación, para evitar sesgos de nuevos códigos diagnósticos. Es por esta razón que la medición de consumo de medicamentos se realiza a través de la medida de DDD.

Para valorar el cambio, se elaboró una escala a partir de la definición de cinco intervalos, según el nivel de cambio porcentual anual medido en unidades dispensadas, entre los periodos analizados (tabla 1 ).

De esta manera, se realizaron análisis mediante cambios porcentuales, tomando como referencia el periodo 2010-2011, como antes de la actualización, y el año 2012 como posterior a esta. Se realizaron análisis por canales de distribución (institucional y comercial) $\mathrm{y}$, además, se compararon las tecnologías seleccionadas con sus respectivos comparadores clínicos. Es necesario tener en cuenta

TABLA 1. TeCnOLOGías UTILIZADAS EN EL ANÁLISIS

\begin{tabular}{|c|c|c|}
\hline Principio activo & Concentración & Comparador \\
\hline Atorvastatina & $10 \mathrm{mg}, 20 \mathrm{mg}, 40 \mathrm{mg}$ & Lovastatina \\
\hline Bosentán & $12,5 \mathrm{mg}$ y $62,5 \mathrm{mg}$ & Amlodipino \\
\hline Capecitabina & $500 \mathrm{mg}$ & \\
\hline Dinoprostona & $10 \mathrm{mg}$ & \\
\hline Etanercept & $25 \mathrm{mg} 50 \mathrm{mg}$ & \\
\hline Imatinib & $100 \mathrm{mg} 400 \mathrm{mg}$ & \\
\hline Latanoprost & $0,05 \mathrm{mg} / \mathrm{ml}$ & Timolol \\
\hline Olanzapina & $10 \mathrm{mg}$ y $5 \mathrm{mg}$ & Haloperidol \\
\hline Rituximab & $10 \mathrm{mg} / \mathrm{ml}$ & \\
\hline Trastuzumab & $440 \mathrm{mg}$ & \\
\hline Valaciclovir & $1000 \mathrm{mg}$ y $500 \mathrm{mg}$ & Aciclovir \\
\hline Tenofovir-emtricitabina & $300 \mathrm{mg}$ y $200 \mathrm{mg}$ & \\
\hline Labetalol & $100 \mathrm{mg} / \mathrm{ml}$ & Hidralazina \\
\hline Doxazosina & $2 \mathrm{mg}$ y $4 \mathrm{mg}$ & Prazosina \\
\hline Alteplasa & $50 \mathrm{mg}$ & \\
\hline Cefuroxima & $250 \mathrm{mg}$ y $500 \mathrm{mg}$ & \\
\hline Gemcitabina & $1 \mathrm{~g} \mathrm{y} 200 \mathrm{mg}$ & \\
\hline Sertralina & $25 \mathrm{mg}, 50 \mathrm{mg}$ y $100 \mathrm{mg}$ & Fluoxetina \\
\hline Micofenolato & $180 \mathrm{mg}, 250 \mathrm{mg}, 360 \mathrm{mg}$ y $500 \mathrm{mg}$ & Ciclosporina \\
\hline Carvedilol & $12,5 \mathrm{mg}, 6,25 \mathrm{mg}$ y $25 \mathrm{mg}$ & Metoprolol \\
\hline
\end{tabular}


que las tecnologías fueron agrupadas con el fin de obtener unidades totales a cada tipo de presentación y con independencia de si es tecnología original o copia, ya que lo que se busca evaluar es el consumo total de los principios activos.

La investigación parte de los siguientes supuestos:

1. El Sismed asegura el registro de la totalidad de las transacciones del mercado, así este sea cubierto o no por el sistema de salud mediante el Pos. De tal manera que el total de unidades despachadas en un periodo corresponde al total accedido por la población dentro del territorio colombiano. Así, un incremento en el consumo de una tecnología específica, implica una respuesta positiva efectiva a una mayor demanda de la población que accedió a ella.

2. Ante el cambio, el asegurador (EPS) entiende la existencia de diferentes y mejores opciones de atención ante la enfermedad y puede brindarle a la población mejores condiciones, en especial a aquellos sectores con dificultades económicas, que de manera ineludible requieren el respaldo del Estado para el acceso a las tecnologías. Con lo anterior, se parte del hecho de que el aumento de la demanda de tecnologías es entendido y amparado por el asegurador en salud.
Se espera que las NTS presenten un incremento o se mantengan igual en su participación global de mercado. Si se presentan grandes incrementos en su demanda, ello significaría una mejora en el acceso y consolidaría la idea de que era necesario actualizar el pos de manera apremiante. De esta manera, las tecnologías denominadas comparadores deberían ser objeto de reemplazo, es decir, se tendrían que estancar en su crecimiento o disminuir su cantidad de dispensación. De igual manera, si el crecimiento en la demanda era, como efecto, de cobertura dentro del sistema, el mayor cambio se debería reflejar dentro del canal institucional.

El no cambio en las cantidades totales, sino en la distribución de dispensación en los canales, se entendería como un efecto de cambio de responsabilidades; es decir, previamente al sistema, las personas accedían por gasto de bolsillo o por los mecanismos de excepción, y ahora lo hacen a través del sistema, si hablamos de un cambio de dispensación de un canal comercial hacia uno institucional. Por su parte, la disminución absoluta de las cantidades solo podría explicarse por una transformación de la demanda hacia otras tecnologías ahora excluidas o por otros efectos dentro del sistema, en donde la inclusión dentro del modelo regulado implica ahora su uso racional.

Además de evaluar el acceso, se identificaron dos aspectos que pueden ser determinantes en los cambios de demanda: 1) la transforma-

TABLA 2. ESCALA DE RANGOS POR CAMBIOS PORCENTUALES DE UNIDADES DISPENSADAS ENTRE LOS PERIODOS ANALIZADOS

\begin{tabular}{|c|c|c|}
\hline Descripción & Rango de cambio & Nomenclatura \\
\hline Disminución importante & $\leq-25 \%$ & - \\
\hline Disminución & Entre -25 y $0 \%$ & - \\
\hline Aumento & Entre 0 y $50 \%$ & + \\
\hline Aumento considerable & Entre 50 y $100 \%$ & ++ \\
\hline Aumento importante & Aumento en más del $100 \%$ & +++ \\
\hline
\end{tabular}

Fuente: elaboración propia 
ción de la demanda entre marcas originales, normalmente oligopólicas, y copias; y 2) los cambios en el precio mediano neto por unidad de medida de las tecnologías. Este último dato asume el sesgo de menor calidad reportado dentro del Sismed. También se realizó un análisis de precios por unidad mínima de las tecnologías analizadas (precio por miligramo), mediante la metodología de cambio porcentual del 2011 al 2012, incluyendo a las tecnologías que sufrieron algún tipo de regulación por parte del Ministerio de Salud y Protección Social.

\section{Resultados}

De las 20 tecnologías analizadas, 18 (90\%) presentaron incremento en el consumo en el periodo 2010-2012. En este mismo lapso, un $60 \%$ de las tecnologías incorporadas presentaron un incremento en el consumo de más del $100 \%$ en sus unidades despachadas (tabla 3) y un $15 \%$ presentó crecimientos entre 50 y $100 \%$, siendo mayor el incremento a nivel de canal institucional. En contraste, solo un $10 \%$ de las tecnologías nuevas incluidas redujo su participación de mercado en el periodo 2010-2012.

En el periodo 2011-2012 (tabla 3) el 75\% de las tecnologías presentaron incremento en sus unidades dispensadas, el $50 \%$ de estas en más del doble con relación al periodo anterior. De igual manera, el $20 \%$ creció entre 0 y $50 \%$, y solo cinco tecnologías mostraron disminuciones de uso en este lapso. Como referencia de análisis se toma el incremental del 2010 al 2012, ya que en el 2011 se presentaron alzas generalizadas en el uso de la mayoría de tecnologías; por ende, la medida de corte del 2010, antes de la actualización, refleja un cambio significativo en el uso real de tecnologías.
A pesar de que no fue tomada la posible incidencia de una enfermedad en el incremento del consumo de tecnologías, cabe tener en cuenta que estas en su mayoría presentaron un aumento de su dispensación en más del $100 \%$, algo que está por encima de cualquier incidencia de enfermedades. Resulta categórico el hecho de que solo el $10 \%$ de las tecnologías analizadas presentaron reducción en su dispensación, lo que indica que sí eran necesarias para la atención de la población.

La mayoría de las tecnologías que tenían comparador dentro del pos también presentaron incrementos en su uso en el periodo de implementación del Acuerdo 029 del 2011. No obstante, se destaca el decrecimiento de haloperidol frente a olanzapina, el cual disminuyó su uso en más del $25 \%$. El no contar con número de pacientes se convierte en un talón de Aquiles para este análisis, ya que puede desconocerse el impacto de incidencias sobre enfermedades tratadas con las tecnologías analizadas.

Cabe destacar que no se realizó la comparación de labetalol con hidralazina, ya que de esta última no se encontraron unidades dispensadas en el Sismed.

En la tabla 5 se describen los cambios entre los diferentes canales de distribución de medicamentos en los años estudiados. Se evidencia que la mayoría de las tecnologías que crecieron en más del $100 \%$ entre el 2010 y el 2012, tienen gran participación, tanto en el canal comercial como en el institucional. Sin embargo, en el canal institucional solo el $5 \%$ presentó decrecimiento en participación.

Además, en ambos canales se experimentó un crecimiento del uso de las tecnologías en el periodo $2010-2012$, de 80 y $90 \%$ en los canales comercial e institucional, respectivamente. La distribución por canales identifica que el crecimiento en la participación de las tecno- 

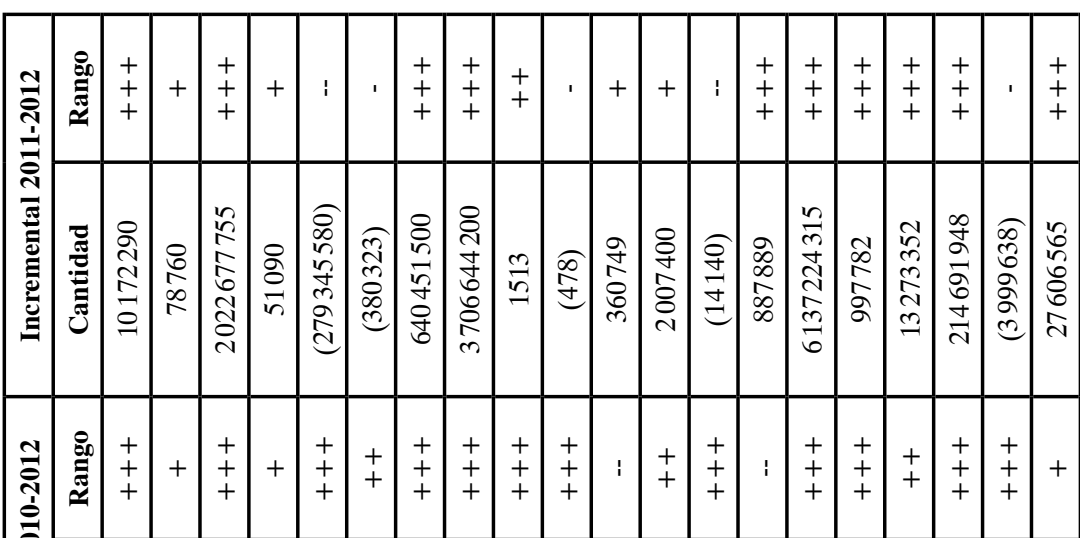

告

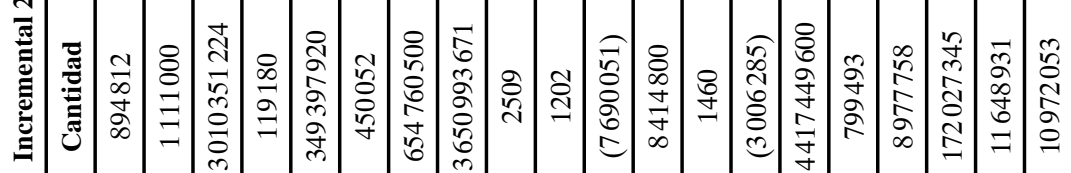

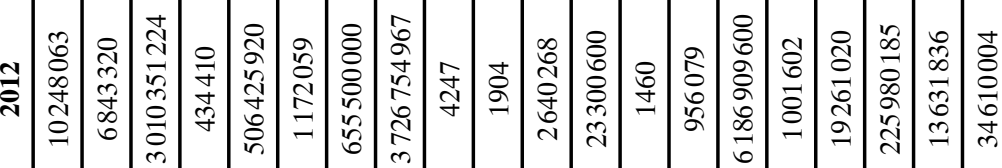

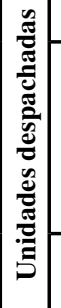

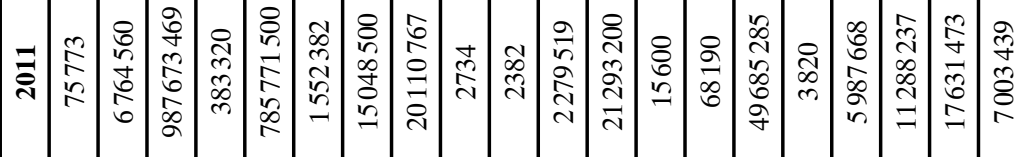

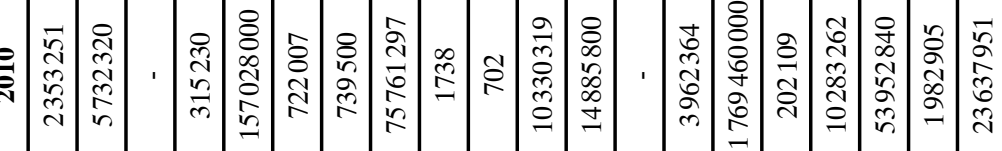

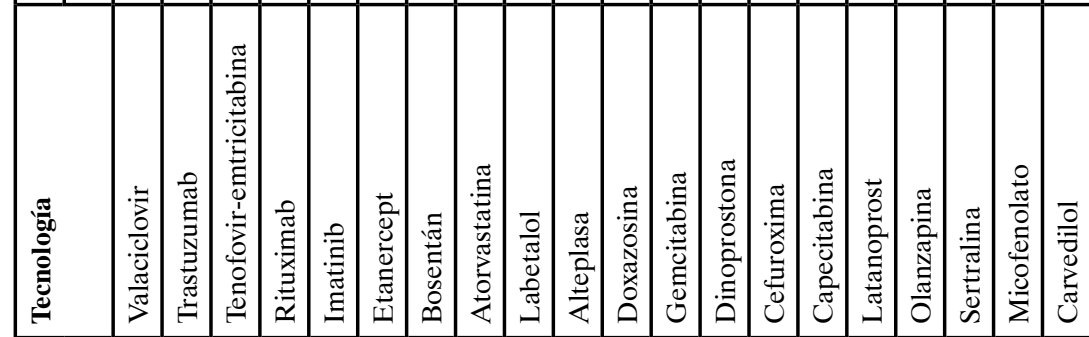


TABLA 4. COMPORTAMiEnto de las tecnOlogías CON COMPARADORES POS, 2010-2012

\begin{tabular}{|l|l|c|c|c|c|c|c|}
\hline & & \multicolumn{3}{|c|}{ Incremental 2010-2012 } & \multicolumn{3}{c|}{ Incremental 2011-2012 } \\
\cline { 5 - 10 } $\begin{array}{l}\text { Tecnología } \\
\text { nueva Pos }\end{array}$ & $\begin{array}{l}\text { Comparador } \\
\text { antiguo Pos }\end{array}$ & $\begin{array}{c}\text { Rango } \\
\text { nuevo } \\
\text { Pos }\end{array}$ & $\begin{array}{c}\text { Incremental } \\
\text { cantidades } \\
\text { antiguo Pos }\end{array}$ & $\begin{array}{c}\text { Rango } \\
\text { antiguo } \\
\text { Pos }\end{array}$ & $\begin{array}{c}\text { Rango } \\
\text { nuevo } \\
\text { Pos }\end{array}$ & $\begin{array}{c}\text { Incremental } \\
\text { cantidades } \\
\text { antiguo Pos }\end{array}$ & $\begin{array}{c}\text { Rango } \\
\text { antiguo } \\
\text { Pos }\end{array}$ \\
\hline Valaciclovir & Aciclovir & +++ & 8892282 & +++ & +++ & 12984395 & +++ \\
\hline Bosentán & Amlodipino & +++ & 4146061977 & +++ & +++ & 4210513295 & +++ \\
\hline Micofenolato & Ciclosporina & +++ & 905760 & +++ & - & 974738 & +++ \\
\hline Sertralina & Fluoxetina & +++ & 528910420 & +++ & +++ & 269374629 & ++ \\
\hline Olanzapina & Haloperidol & ++ & 29615240 & ++ & +++ & $(147716953)$ & - \\
\hline Atorvastatina & Lovastatina & +++ & 2481626960 & +++ & +++ & 2818034206 & +++ \\
\hline Carvedilol & Metroprolol & + & 560818466 & +++ & +++ & 568493010 & +++ \\
\hline Doxazosina & Prazosina & -- & 12776801 & ++ & + & 12582110 & ++ \\
\hline Latanoprost & Timolol & +++ & 1040964 & +++ & +++ & 1037630 & +++ \\
\hline
\end{tabular}

Fuente: elaboración propia

TABLA 5. VARIACIONES EN CANTIDADES DESPACHADAS TOTALES POR CANAL DE DISTRIBUCIÓN

\begin{tabular}{|c|c|c|c|c|c|c|}
\hline \multirow[b]{2}{*}{ Tecnología } & \multicolumn{3}{|c|}{ Incremental 2010-2012 } & \multicolumn{3}{|c|}{ Incremental 2011-2012 } \\
\hline & $\begin{array}{c}\text { Canal } \\
\text { institucional }\end{array}$ & $\begin{array}{c}\text { Canal } \\
\text { comercial }\end{array}$ & Total & $\begin{array}{c}\text { Canal } \\
\text { institucional }\end{array}$ & $\begin{array}{c}\text { Canal } \\
\text { comercial }\end{array}$ & Total \\
\hline 1. Valaciclovir & $(306306,87)$ & 8201119,30 & +++ & 23857,77 & 10148432,30 & +++ \\
\hline 2. Trastuzumab & 207240,00 & 903760,00 & + & $(14520,00)$ & 93280,00 & + \\
\hline $\begin{array}{l}\text { 3. Tenofovir- } \\
\text { emtricitabina }\end{array}$ & 2195265306 & 815085918,37 & +++ & 1242673469,39 & 780004285,71 & +++ \\
\hline 4. Rituximab & 30050,00 & 89130,00 & + & 210,00 & 50880,00 & + \\
\hline 5. Imatinib & 223637280,00 & 125760640,00 & +++ & $(377984860,00)$ & 98639280,00 & -- \\
\hline 6. Etanercept & 94167,86 & 355883,86 & ++ & $(459917,86)$ & 79594,57 & - \\
\hline 7. Bosentán & 470282000 & 184478500 & +++ & 456406250,00 & 184045250,00 & +++ \\
\hline 8. Atorvastatina & 128134625 & 3522859045,50 & +++ & 125287195,93 & 3581357003,91 & +++ \\
\hline 9. Labetalol & 586,57 & 1922,78 & +++ & $(262,18)$ & 1774,78 & ++ \\
\hline 10. Alteplasa & 1136,50 & 65,50 & +++ & 488,50 & $(966,00)$ & - \\
\hline 11. Doxazosina & 1268007,06 & $(8958058,04)$ & -- & $(10647,92)$ & 371396,46 & + \\
\hline 12. Gemcitabina & 8827000 & $(412200,00)$ & ++ & 4407000,00 & $(2399600,00)$ & + \\
\hline 13. Dinoprostona & 1460 & - & +++ & $(14140,00)$ & - & -- \\
\hline 14. Cefuroxima & 147580,58 & $(3153865,50)$ & -- & 416537,40 & 471352,00 & +++ \\
\hline 15. Capecitabina & 2117593200 & 2299856400 & +++ & 3330139950 & 2807084365 & +++ \\
\hline 16. Latanoprost & 2466,95 & 797026,50 & +++ & 9283,80 & 988498,23 & +++ \\
\hline 17. Olanzapina & 6342896,22 & 2634862,02 & ++ & 2519089,96 & 10754261,80 & +++ \\
\hline 18. Sertralina & 19837093,29 & 152190252,00 & +++ & 24313757,27 & 190378190,60 & +++ \\
\hline 19. Micofenolato & 6659518,68 & 4989412,55 & +++ & $(8979194,48)$ & 4979556,84 & - \\
\hline 20. Carvedilol & 9805059,91 & 1166992,68 & + & 5745566,54 & 21860998,38 & +++ \\
\hline
\end{tabular}

Fuente: elaboración propia 
logías, vía mayor preferencia de los usuarios, es indistinto tanto del canal comercial como del institucional. Asimismo, la participación de las tecnologías que crecen a más del doble se apalanca en aumentos considerables en el canal institucional, tomando en cuenta que el $55 \%$ de estas crecen en dicho canal en el periodo 2010-2012, y este canal distribuye la mayor parte de medicamentos consumidos en hospitales y centros de atención de pacientes en general. De esta manera, se percibe cómo el aumento del consumo de dichas tecnologías pasó por los distintos niveles de complejidad, ya que se presentan aumentos tanto en tecnologías de gasto de bolsillo como en las utilizadas exclusivamente en el contexto intrahospitalario, las cuales se consumen a través del canal institucional.

Sobre el al análisis realizado al comportamiento de los precios de las tecnologías (tabla 6), se parte de la hipótesis de que los costos de estas no deben constituir un factor determinante para su uso, ya que para los profesionales de la salud esto debe ser indiferente.

El análisis comparativo de costos medianos mostró que un total de 12 de las tecnologías evaluadas sufrieron disminución de precios entre los años 2011 y 2012. Esta fue una

TABLA 6. Costo MEdio POR Miligramo de LAS TECNOLOGías EVALUAdAS

\begin{tabular}{|c|c|c|c|c|}
\hline \multirow{2}{*}{ Tecnologías } & \multicolumn{2}{|c|}{ Precio medio por miligramo } & \multirow{2}{*}{$\begin{array}{c}\text { Incremento porcentual } \\
\text { 2011-2012 }\end{array}$} & \multirow{2}{*}{ Rango } \\
\hline & 2011 & 2012 & & \\
\hline Atorvastatina $(\varepsilon)$ & 163,35 & 152,380017 & $-6,72$ & + \\
\hline Bosentán & $2443,11^{*}$ & 1874,97 & $-23,29$ & - \\
\hline Capecitabina & 32,5 & 28,3631 & $-12,73$ & - \\
\hline Dinoprostona & & 223186,86 & - & \\
\hline Etanercept $(\varepsilon)$ & 15348,06 & 14033,27 & $-8,57$ & - \\
\hline Imatinib $(\varepsilon)$ & 200,22 & 197,72 & $-1,25$ & - \\
\hline Latanoprost & 31306,5767 & 19954,64 & $-36,26$ & -- \\
\hline Olanzapina & 988,91 & 2032,49 & 105,53 & +++ \\
\hline Rituximab & 7958,40 & 9058,46 & 13,82 & + \\
\hline Trastuzumab $(\varepsilon)$ & 12606,9 & 12572,92 & $-0,27$ & - \\
\hline Valaciclovir & 15,11 & 11,3221 & $-25,07$ & - \\
\hline Tenofovir-emtricitabina & 49,8876404 & 30,2 & $-39,46$ & -- \\
\hline Labetalol & 379,78 & & $-100,00$ & -- \\
\hline Doxazosina & 997,99 & 1483,1 & 48,61 & + \\
\hline Alteplasa & 48174,95 & 45567,2691 & $-5,41$ & - \\
\hline Cefuroxina & 8,32 & 9,3234 & 12,06 & + \\
\hline Gemcitabina $(\varepsilon)$ & 164,8 & 198,7031 & 20,57 & + \\
\hline Sertralina & 54,24 & 43,98 & $-18,92$ & - \\
\hline Micofenolato $(\varepsilon)$ & 15,66 & 16,32 & 4,21 & + \\
\hline Carvedilol & 63,51 & 59,97 & $-5,57$ & - \\
\hline
\end{tabular}

* Es el precio del 2010, dada la inexistencia de este valor para 2011.

( $\varepsilon$ Estos medicamentos fueron regulados el 30 de agosto del 2012 por el Ministerio de Salud y Protección Social. Fuente: elaboración propia 
tendencia general en el mercado de medicamentos en Colombia, como producto de la entrada de competidores y la regulación de precios que se llevó a cabo en el 2012.

Se destaca que tecnologías como la doxazosina y la cefuroxima presentaron aumentos significativos en su precio y, al mismo tiempo, disminución en sus cantidades dispensadas del 2010 al 2012. De esta manera, se observa cómo el mercado de tecnologías logra funcionar parcialmente para algunas de ellas, pese a las fallas naturales propias de los mercados de la salud. La integración de tecnologías en el plan de beneficios, las saca de una zona gris donde el prestador recobra, para pasar a ser unas tecnologías que entran en la dinámica del mercado, con tendencia decreciente de los precios debido a la competencia, incluso por debajo de su precio de regulación. Solo seis de las tecnologías evaluadas fueron sometidas a regulación de precios. Sin embargo, la mayoría de las tecnologías del análisis disminuyeron su precio, bajo la hipótesis de que la entrada al mercado (plan de beneficios) las somete a la competencia, debido a que el prestador asume el costo de esta.

\section{Discusión}

El presente estudio revela cambios en el comportamiento de la demanda de servicios (nuevas tecnologías), producto de la actualización del pos (unificación de los planes de beneficios y actualización del listado de tecnologías) en el sGsss de Colombia. Tal como se esperaba, los resultados muestran que la actualización del plan de beneficios mediante el Acuerdo 029 de 2011 representa un mayor acceso de los pacientes a las nuevas tecnologías, las cuales en algunos de los casos reemplazaron tecnologías con la misma indicación que se utilizaban hace más de diez años dentro del pos. Sin embargo, es necesario tener en cuenta que el desconocimiento del número de pacientes por patología crea un sesgo en la identificación del impacto neto del acuerdo de actualización del plan de beneficios.

El comportamiento de la dispensación de tecnologías (medicamentos) puede medir en forma indirecta los cambios del acceso a los servicios de salud. Sin embargo, existen muchos factores que pueden influir en el comportamiento de la dispensación, el principal son las tasas de prevalencia e incidencia de las enfermedades. Estos efectos pueden neutralizarse si se analiza el comportamiento de las tecnologías que se encontraban en el plan de beneficios antes del Acuerdo 29 de 2011. De esta manera, la disminución en el uso de algunas de estas tecnologías quizá refleje un decrecimiento en las preferencias de los usuarios.

De igual manera, es posible que el efecto de cobertura dentro de la prima genere un efecto de mayor consumo de estas nuevas tecnologías como resultado del aseguramiento. El uso de los medicamentos se da como respuesta a una intervención en salud, y aunque podría generar algún efecto de inducción a la demanda, se esperaría que el efecto sea únicamente de mejor oportunidad frente a los disponibles por parte del cuadro médico.

Por otra parte, los aumentos reportados dejan en duda el efecto que puedan tener accesos extraordinarios como la tutela o los comités técnico-científicos, que se prevén como la opción para aquellas tecnologías que, si bien son necesarias, están excluidas del Pos.

La falta de actualización del plan de beneficios colombiano, es la mejor explicación del desplazamiento y el reemplazo lento, pero progresivo, que las nuevas tecnologías incluidas en el pos provocan en las tecnologías anteriores al acuerdo, como lo reflejan los resultados de esta primera aproximación. De igual manera, como indicador de mejor acceso, se espera una reducción en el número 
de tutelas y comités técnico-científicos para la solicitud de procedimientos y tecnologías en el país, ya que el número de tutelas solicitando tecnologías no pos ha sido muy alto y ha generado que el Estado, a través del Fosyga, haya pagado en el año 2009 más de dos billones de pesos a las EPs por concepto de los recobros no pos (9).

El progreso de la enfermedad en los pacientes tratados con las tecnologías de análisis no fue tenido en cuenta. Sin embargo, esta limitación también es cubierta con el análisis de comportamiento de los comparadores con la misma indicación de las tecnologías analizadas.

Muchas de las inclusiones en el pos 2012 se realizaron por iniciativa de la CRES, la cual se basó en estudios de evaluación de tecnologías en salud contratados. Esto representa un avance en la estandarización de criterios técnicos de priorización, como parte del proceso integral de la toma de decisiones (10). Cabe destacar que, teniendo en cuenta otras evaluaciones de la actualización, las cantidades dispensadas de tecnologías pueden de igual manera estar afectadas, tanto por la concentración de más servicios quirúrgicos, como por más tecnologías nuevas para los sistemas orgánicos osteomuscular, digestivo y circulatorio. Muchas de las tecnologías nuevas, fruto de la actualización, se concentran en dichos sistemas orgánicos, que son los que la mayoría de estudios consideran de más impacto para la población colombiana.

Cabe aclarar que en el año 2011 muchos medicamentos fueron sometidos a regulación de precio por parte de las autoridades nacionales. Esa regulación no fue tenida en cuenta en esta investigación, ya que en teoría al estar una tecnología dentro del plan de beneficios, su precio no debe ser tenido en cuenta en la toma de decisiones en el sistema de salud. Además de ello, la utilización de las tecnologías analizadas no depende directamente de los pacientes, sino de los profesionales de la salud encargados de la toma de decisiones, donde el precio de las tecnologías no debería influir en su uso.

Por otra parte, una de las grandes críticas al Sismed ha sido la inconsistencia en el precio, debido a que este tiene sesgos por su falta de claridad cuando se incluyen bonificaciones, descuentos y donaciones, entre otras opciones. Sin embargo, poco se ha criticado con relación a las cantidades reportadas, ya que estas en general tienden a ser un dato más consistente y reflejan la cantidad de unidades circulantes dentro del país.

A pesar de que no se presentaron diferencias considerables en la dispensación de medicamentos, según medio de distribución (comercial e institucional), el seguimiento de dicha distribución puede encontrar diferencias en el futuro, ya que se espera un gran crecimiento de las tecnologías en el canal institucional; este se encuentra vinculado a los hospitales, los cuales son grandes distribuidores. Esta tendencia debe ser marcada, principalmente por el cambio en las preferencias de los médicos y profesionales de salud, si se considera que la nueva tecnología incluida en POs es vanguardista para el tratamiento de enfermedades específicas, en relación con tecnologías antiguas en el pos antes del Acuerdo 29 de 2011.

La actualización del plan de beneficios colombiano le brindó la oportunidad a la mayoría de usuarios del sistema, de acceder con mayor facilidad a un conjunto de nuevas tecnologías. La constante actualización del POS, con base en evaluaciones económicas, evaluaciones de costo-utilidad, impactos presupuestarios o revisiones sistemáticas, son de gran importancia para la mejora constante del desempeño del sistema de salud colombiano, lo que da como resultado 
tanto el mejoramiento del acceso como el de la calidad en la atención.

Teniendo en cuenta que la actualización no solo se realizó para medicamentos, valdría la pena hacer un análisis equivalente para los procedimientos y otras tecnologías, con el fin de extender el análisis a las nuevas actualizaciones y a la persistencia de los efectos identificados, para así evaluar integralmente el proceso.

\section{Conclusión}

La incorporación de las nuevas tecnologías en el 2012 mejoró el acceso a los servicios de salud, entendido como un mayor consumo de estas y el reemplazo de otras en uso desde hace más de diez años. Si bien esto podría ser un efecto del aseguramiento, al ser los medicamentos resultados de una atención médica, este incremento podría entenderse como pacientes efectivos con mayor acceso. El incremento en la dispensación de medicamentos se vio tanto en el canal institucional como en el comercial, lo que da cuenta de que el impacto sobre los pacientes se presenta en todos los niveles de atención. No obstante, se espera que los incrementos sean más marcados en el canal institucional, por mayor uso de las tecnologías por parte de los profesionales de la salud, en virtud de tecnologías nuevas vanguardistas.

En algunos casos, como al comparar haloperidol vs. olanzapina, encontramos que las nuevas tecnologías incluidas en el Acuerdo 029 entraron en alguna medida a reemplazar tecnologías más antiguas en el plan de beneficios. Este reemplazo puede presentarse en varias de las tecnologías evaluadas, pero su impacto se va presentando lentamente a medida que los profesionales trasladen sus preferencias hacia las nuevas tecnologías. Por su parte, el comportamiento del precio por miligramo de las tecnologías utilizadas tendió hacia la baja. Este fenómeno se explica porque la entrada de la tecnología al pos implica que el prestador del servicio de salud asume su costo de la prima UPC, lo que comienza a impactar en las decisiones de uso de tecnologías.

La actualización del plan de beneficios mediante el Acuerdo 29 de 2011 permitió que el acceso a muchos servicios de salud mejorara, lo que a su vez permitió optimizar el desempeño del sGsss.

\section{Referencias bibliográficas}

1. Londono JL, Frenk J. Structured Pluralism: Towards an Innovative Model for Health System Reform in Latin America. Health Policy. 1997 Jul; 41 (1): 1-36.

2. Enthoven A. Theory and Practice of Managed Competition in Health Care Finance. Nueva York: North Holland Publishing Company; 1988.

3. Contreras-Hernández I, Prisco FE, Alvis-Gúzman, Doral S. El uso de evaluación económica para la toma de decisiones en intervenciones oncológicas: la experiencia de México, Colombia y Brasil. PharmacoEconomics Spanish Research Articles. 2012 Nov; 9 (4): 117-33.

4. Ramírez J. La emergencia social en salud: de las contingencias regulatorias a la desilusión ciudadana Gerencia y Políticas de Salud. 2010; 9 (18): 124-143.

5. Gualtero ML. La tutela y el derecho a la salud: Período 2006-2008: equipo investigativo del programa de salud y seguridad social de la Defensoría del Pueblo. Bogotá: Defensoría del Pueblo; 2009.

6. Vélez AL. La acción de tutela: ¿un mecanismo de protección del derecho a la salud y un proceso alterno para acceder a servicios de salud? Colombia Médica. 2005; 36 (3): 199-208.

7. Comisión de Regulación Nacional. Acuerdo número 029 de 2011. Bogotá: Comisión de Regulación Nacional; 2011[Internet] [acceso: 16 de abril del 2014]. Disponible en: http://www. minsalud.gov.co/salud/POS/Documents/sitio/ACTUALIZACI \%C3\%93N\%20POS\%202012/Acuerdo \%20 029\%20de\%202011.pdf

8. Ministerio de la Protección Social. Sistema de Información de Precios de Medicamentos. Bogotá: Ministerio de Industria y Comercio; 2011 [Internet] [acceso: 2013]. Disponible en: http://web.sispro.gov. co/WebPublico/sIsmed/LibroVirtual/index2.html.

9. Arias SJ. ¿Tutelas o comités técnicos científicos, cuál es la verdadera causa del problema? Revista de Derecho Público. 2011; 27: 2-28.

10. Cardona Á., Mejía L, Vargas G, Álvarez E, Nieto E. Análisis de la actualización del Plan Obligatorio de Salud del Sistema de Seguridad Social en Salud colombiano. Rev. Salud Pública. 2013; 15 (2): 258-70. 